\title{
Recurrence relations of Kummer functions and Regge string scattering amplitudes
}

\author{
Department of Electrophysics, National Chiao-Tung \\ University, Hsinchu, Taiwan, R.O.C. and \\ National Center for Theoretical Sciences, Hsinchu, Taiwan, R.O.C.
}

\begin{abstract}
We discover an infinite number of recurrence relations among Regge string scattering amplitudes [10, 23] of different string states at arbitrary mass levels in the open bosonic string theory. As a result, all Regge string scattering amplitudes can be algebraically solved up to multiplicative factors. Instead of decoupling zero-norm states in the fixed angle regime, the calculation is based on recurrence relations and addition theorem of Kummer functions of the second kind. These recurrence relations among Regge string scattering amplitudes are dual to linear relations or symmetries among high-energy fixed angle string scattering amplitudes discovered previously.
\end{abstract}

*Electronic address: jcclee@cc.nctu.edu.tw

${ }^{\dagger}$ Electronic address: yoshihiro.mitsuka@gmail.com 


\section{INTRODUCTION}

There are two high-energy regimes of string scattering amplitudes, the fixed angle regime or Gross Regime (GR) [1, 2], and the fixed momentum transfer regime or Regge regime (RR) [3 3 ]. The scattering amplitudes of these two regimes contain information complementary to each other [10]. Recently a saddle point method was used to calculate string-tree amplitudes in the GR for string states at arbitrary mass levels [11-14]. See also the developments in [15 18]. The ratios of scattering amplitudes among different string states at each fixed mass level in the GR can be extracted and were found to be independent of scattering energy and scattering angle. Alternatively, these infinite number of ratios can be rederived algebraically by solving linear relations or stringy Ward identities based on the calculation of decoupling of zero-norm states (ZNS) [19-21] in the string spectrum. These infinite number of ratios were interpreted as high-energy spacetime symmetry of string theory conjectured by Gross more than twenty years ago [22].

In contrast to the GR, things were less known in the RR. It was known that there is no saddle point method for the calculation of Regge string scattering amplitudes. More recently, a subset of Regge string scattering amplitudes were calculated by a direct method and it was found that all the Regge string scattering amplitudes can be expressed in terms of Kummer functions of the second kind [10, 23]. Moreover, these Regge string scattering amplitudes can be used to reextract the ratios among high-energy fixed angle scattering amplitudes calculated in the GR mentioned above [24, 25]. Presumably, there must be intimate link between string scatterings in the GR and RR. Unlike the case of the GR, the relations among Regge string scattering amplitudes of different string states were not understood and the ratios were suspected to depend on the scattering energy and scattering angle. A natural question is then raised:

Are there any kind of relations among Regge string scattering amplitudes of different string states which are dual to those linear relations [11 14] calculated in the fixed angle regime?

To answer this question, in this paper we first calculate the complete set of Regge string scattering amplitudes which turns out to be much more numerous than those in the GR. We consider four-point function with three tachyons and one most general Regge string state. 
Note that, in order to calculate Regge stringy Ward identities based on decoupling ZNS, one needs to use the complete Regge string scattering amplitudes. We then derive Regge stringy Ward identities for the first few mass levels based on the decoupling of ZNS. We found that, unlike the case for the GR, the Regge stringy Ward identities were not good enough to solve all the Regge string scattering amplitudes algebraically. This is due to the much more numerous Regge string scattering amplitudes than those in the GR at each fixed mass level. However, we found that, for mass levels $M^{2}=2$ and 4, all the Regge stringy Ward identities can be explicitly proved by using a set of identities of Kummer function, namely, the recurrence relations of Kummer functions of the second kind [26]. Presumably, the calculation can be generalized to arbitrary higher mass levels. We then went a step further and show that, instead of Regge stringy Ward identities, one can use these recurrence relations of Kummer functions to solve all Regge string scattering amplitudes at arbitrary higher mass levels algebraically up to multiplicative factors.

Finally, we calculate explicitly some examples of recurrence relations among Regge string scattering amplitudes based on recurrence relations of Kummer functions. These recurrence relations are, in general, independent of Regge stringy Ward identities calculated from decoupling of ZNS. They are thus more general than Regge stringy Ward identities. In addition, as an example, we construct an inter-mass level recurrence relation which was calculated by using addition theorem of Kummer functions [26]. Following the same procedure, one can construct infinite number of recurrence relations among Regge string scattering amplitudes at arbitrary mass levels. The recurrence relations for Regge string scattering amplitudes are reminiscent of BCJ relations [27-31] for Yang-Mills gluon color-stripped scattering amplitudes where kinematic variables show up in the coefficients of the relations.

\section{GR AND RR STRING AMPLITUDES}

We begin with a brief review of high-energy string scattering in the fixed angle regime,

$$
s,-t \rightarrow \infty, t / s \approx-\sin ^{2} \frac{\phi}{2}=\text { fixed }(\text { but } \phi \neq 0)
$$

where $s=-\left(k_{1}+k_{2}\right)^{2}, t=-\left(k_{2}+k_{3}\right)^{2}$ and $u=-\left(k_{1}+k_{3}\right)^{2}$ are the Mandelstam variables and $\phi$ is the center of mass scattering angle. It was shown [13, 14] that for the 26D open bosonic string the only states that will survive the high-energy limit at mass level $M_{2}^{2}=2(N-1)$ 
are of the form (we choose the second state of the four-point function to be the higher spin string state)

$$
|N, 2 m, q\rangle \equiv\left(\alpha_{-1}^{T}\right)^{N-2 m-2 q}\left(\alpha_{-1}^{L}\right)^{2 m}\left(\alpha_{-2}^{L}\right)^{q}|0, k\rangle,
$$

where the polarizations of the 2 nd particle with momentum $k_{2}$ on the scattering plane were defined to be $e^{P}=\frac{1}{M_{2}}\left(E_{2}, \mathrm{k}_{2}, 0\right)=\frac{k_{2}}{M_{2}}$ as the momentum polarization, $e^{L}=\frac{1}{M_{2}}\left(\mathrm{k}_{2}, E_{2}, 0\right)$ the longitudinal polarization and $e^{T}=(0,0,1)$ the transverse polarization which lies on the scattering plane. $\eta_{\mu \nu}=\operatorname{diag}(-1,1,1)$. In Eq.(2.2), $N, m$ and $q$ are non-negative integers and $N \geq 2 m+2 q$. These integers characterise the mass square and "spin" of the higher string states. Note that $e^{P}$ approaches to $e^{L}$ in the GR [12]. So we did not put $e^{P}$ components in Eq.(2.2). For simplicity, we choose the particles associated with momenta $k_{1}, k_{3}$ and $k_{4}$ to be tachyons. It turned out that the high-energy fixed angle scattering amplitudes can be calculated by using the saddle-point method [13] to be

$$
\mathcal{T}^{(N, 2 m, q)}=\sqrt{\frac{2 \pi}{K f_{0}^{\prime \prime}}} e^{-K f_{0}}\left[(-1)^{N-q} \frac{2^{N-q-2 m}(2 m) !}{m ! M_{2}^{q+2 m}} \tau^{-\frac{N}{2}}(1-\tau)^{\frac{3 N}{2}} E^{N}+O\left(E^{N-2}\right)\right]
$$

where $K \equiv-k_{1} \cdot k_{2} \rightarrow 2 E^{2}, f(x) \equiv \ln x-\tau \ln (1-x), \tau \equiv-\frac{k_{2} \cdot k_{3}}{k_{1} \cdot k_{2}} \rightarrow \sin ^{2} \frac{\phi}{2}$, and the saddlepoint for the integration of moduli, $x=x_{0}=\frac{1}{1-\tau}$, is defined by $f^{\prime}\left(x_{0}\right)=0$. The complete ratios among high-energy fixed angle string scattering amplitudes of different string states at each fixed mass level can be easily calculated from Eq.(2.3) to be [13]

$$
\frac{T^{(N, 2 m, q)}}{T^{(N, 0,0)}}=\left(-\frac{1}{M_{2}}\right)^{2 m+q}\left(\frac{1}{2}\right)^{m+q}(2 m-1) ! !
$$

Alternatively, it was important to discover that [13, 14] the ratios can be calculated by the method of decoupling of two types of ZNS

$$
\text { Type I : } L_{-1}|x\rangle \text {, where } L_{1}|x\rangle=L_{2}|x\rangle=0, L_{0}|x\rangle=0 \text {; }
$$

$$
\text { Type II : }\left(L_{-2}+\frac{3}{2} L_{-1}^{2}\right)|\widetilde{x}\rangle, \text { where } L_{1}|\widetilde{x}\rangle=L_{2}|\widetilde{x}\rangle=0,\left(L_{0}+1\right)|\widetilde{x}\rangle=0 .
$$

in the old covariant first quantized string spectrum. The key step was to use the decoupling of high-energy fixed angle ZNS

$$
\begin{aligned}
L_{-1}|n-1,2 m-1, q\rangle & \simeq M|n, 2 m, q\rangle+(2 m-1)|n, 2 m-2, q+1\rangle, \\
L_{-2}|n-2,0, q\rangle & \simeq \frac{1}{2}|n, 0, q\rangle+M|n, 0, q+1\rangle
\end{aligned}
$$


to deduce the ratios of all amplitudes at the leading order energy in Eq.(2.4). Since the decoupling of ZNS applies to all string loop order, the ratios calculated in Eq.(2.4) were valid to all string loop order and were interpreted as high-energy spacetime symmetry of string theory conjectured by Gross in 1988 [22].

Another high-energy regime of string scattering amplitudes, which contains dual information of the theory, is the fixed momentum transfer regime or RR. That is in the kinematic regime

$$
s \rightarrow \infty,-t=\text { fixed (but }-t \neq \infty \text { ). }
$$

It was found [10] that the number of high energy scattering amplitudes for each fixed mass level in this regime is much more numerous than that of fixed angle regime calculated previously. The complete leading order high-energy open string states in the Regge regime at each fixed mass level $N=\sum_{n, m, l>0} n p_{n}+m q_{m}+l r_{l}$ are

$$
\left|p_{n}, q_{m}, r_{l}\right\rangle=\prod_{n>0}\left(\alpha_{-n}^{T}\right)^{p_{n}} \prod_{m>0}\left(\alpha_{-m}^{P}\right)^{q_{m}} \prod_{l>0}\left(\alpha_{-l}^{L}\right)^{r_{l}}|0, k\rangle .
$$

The case for $q_{m}=0$ has been calculated previously in [10, 23]. We stress that the inclusion of both $\alpha_{-m}^{P}$ and $\alpha_{-l}^{L}$ operators in Eq.(2.10) will be crucial to study Regge string Ward identities to be discussed in the later part of the paper. The momenta of the four particles on the scattering plane are

$$
\begin{aligned}
& k_{1}=\left(+\sqrt{p^{2}+M_{1}^{2}},-p, 0\right), \\
& k_{2}=\left(+\sqrt{p^{2}+M_{2}^{2}},+p, 0\right), \\
& k_{3}=\left(-\sqrt{q^{2}+M_{3}^{2}},-q \cos \phi,-q \sin \phi\right), \\
& k_{4}=\left(-\sqrt{q^{2}+M_{4}^{2}},+q \cos \phi,+q \sin \phi\right)
\end{aligned}
$$

where $p \equiv|\tilde{\mathrm{p}}|, q \equiv|\tilde{\mathrm{q}}|$ and $k_{i}^{2}=-M_{i}^{2}$. The relevant kinematics are

$$
\begin{aligned}
& e^{P} \cdot k_{1} \simeq-\frac{s}{2 M_{2}}, \quad e^{P} \cdot k_{3} \simeq-\frac{\tilde{t}}{2 M_{2}}=-\frac{t-M_{2}^{2}-M_{3}^{2}}{2 M_{2}} ; \\
& e^{L} \cdot k_{1} \simeq-\frac{s}{2 M_{2}}, \quad e^{L} \cdot k_{3} \simeq-\frac{\tilde{t}^{\prime}}{2 M_{2}}=-\frac{t+M_{2}^{2}-M_{3}^{2}}{2 M_{2}} ;
\end{aligned}
$$

and

$$
e^{T} \cdot k_{1}=0, \quad e^{T} \cdot k_{3} \simeq-\sqrt{-t}
$$


where $\tilde{t}$ and $\tilde{t}^{\prime}$ are related to $t$ by finite mass square terms

$$
\tilde{t}=t-M_{2}^{2}-M_{3}^{2}, \tilde{t}^{\prime}=t+M_{2}^{2}-M_{3}^{2}
$$

Note that, unlike the case of GR, here $e^{P}$ does not approach to $e^{L}$ in the RR. The Regge string scattering amplitudes can then be explicitly calculated to be

$$
\begin{aligned}
& A(s, t) \simeq \int_{0}^{1} d y y^{k_{1} k_{2}}(1-y)^{k_{2} k_{3}} \cdot \prod_{n}\left[-\frac{(n-1) ! e^{T} \cdot k_{1}}{(-y)^{n}}-\frac{(n-1) ! e^{T} \cdot k_{3}}{(1-y)^{n}}\right]^{p_{n}} \\
& \prod_{m}\left[\frac{(m-1) ! e^{P} \cdot k_{1}}{(-y)^{m}}+\frac{(m-1) ! e^{P} \cdot k_{3}}{(1-y)^{m}}\right]^{q_{m}} \\
& \prod_{l}\left[-\frac{(l-1) ! e^{L} \cdot k_{1}}{(-y)^{l}}-\frac{(l-1) ! e^{L} \cdot k_{3}}{(1-y)^{l}}\right]^{r_{l}} \\
& \approx \int_{0}^{1} d y y^{-\frac{s}{2}+N-2}(1-y)^{-\frac{t}{2}+N-2} \\
& \cdot \prod_{n>0}\left[\frac{(n-1) ! \sqrt{-t}}{(1-y)^{n}}\right]^{p_{n}} \cdot \prod_{m>1}\left[-\frac{(m-1) ! \frac{\tilde{t}}{2 M_{2}}}{(1-y)^{m}}\right]^{q_{m}} \cdot \prod_{l>1}\left[\frac{(l-1) ! \frac{\tilde{t}^{\prime}}{2 M_{2}}}{(1-y)^{l}}\right]^{r_{l}} \\
& \cdot\left[\frac{\frac{s}{2 M_{2}}}{y}-\frac{\frac{\tilde{t}}{2 M_{2}}}{(1-y)}\right]^{q_{1}}\left[-\frac{\frac{s}{2 M_{2}}}{y}+\frac{\frac{\tilde{t}^{\prime}}{2 M_{2}}}{(1-y)}\right]^{r_{1}} \\
& =\prod_{n>0}[(n-1) ! \sqrt{-t}]^{p_{n}} \cdot \prod_{m>1}\left[-(m-1) ! \frac{\tilde{t}}{2 M_{2}}\right]^{q_{m}} \cdot \prod_{l>1}\left[(l-1) ! \frac{\tilde{t}^{\prime}}{2 M_{2}}\right]^{r_{l}} \\
& \cdot \sum_{i, j}\left(\begin{array}{c}
q_{1} \\
i
\end{array}\right)\left(\begin{array}{c}
r_{1} \\
j
\end{array}\right)\left(-\frac{s}{\tilde{t}}\right)^{i}\left(-\frac{s}{\tilde{t}^{\prime}}\right)^{j} B\left(-\frac{s}{2}+N-1-i-j,-\frac{t}{2}-1+i+j\right) \text {. }
\end{aligned}
$$

In the second equality of the above equation, we have dropped the first term in the bracket with power of $p_{n}$, and the first terms in the brackets with powers of $q_{m}$ and $r_{l}$ for $m, l>1$. These terms lead to subleading order terms in energy in the Regge limit [10, 23]. Now the beta function in Eq.(2.19) can be approximated in the RR by [10, 23]

$$
B\left(-\frac{s}{2}+N-1-i-j,-\frac{t}{2}-1+i+j\right)=B\left(-\frac{s}{2}-1,-\frac{t}{2}-1\right)\left(-\frac{s}{2}\right)^{-i-j}\left(-\frac{t}{2}-1\right)_{i+j}
$$

where $(a)_{j}=a(a+1)(a+2) \ldots(a+j-1)$ is the Pochhammer symbol. Finally we arrive at 
the amplitude with two equivalent expressions

$$
\begin{aligned}
A(s, t)= & \prod_{n>0}\left[(n-1) ! \sqrt{-t}^{p_{n}} \cdot \prod_{m>0}\left[-(m-1) ! \frac{\tilde{t}}{2 M}\right]^{q_{m}} \cdot \prod_{l>1}\left[(l-1) ! \frac{\tilde{t}^{\prime}}{2 M}\right]^{r_{l}}\right. \\
& \cdot B\left(-\frac{s}{2}-1,-\frac{t}{2}+1\right)\left(\frac{1}{M}\right)^{r_{1}} \\
& \cdot \sum_{i=0}^{q_{1}}\left(\begin{array}{c}
q_{1} \\
i
\end{array}\right)\left(\frac{2}{\tilde{t}}\right)^{i}\left(-\frac{t}{2}-1\right)_{i} U\left(-r_{1}, \frac{t}{2}+2-i-r_{1}, \frac{\tilde{t}^{\prime}}{2}\right) \\
= & \prod_{n>0}[(n-1) ! \sqrt{-t}]^{p_{n}} \cdot \prod_{m>1}\left[-(m-1) ! \frac{\tilde{t}}{2 M}\right]^{q_{m}} \cdot \prod_{l>0}\left[(l-1) ! \frac{\tilde{t}^{\prime}}{2 M}\right]^{r_{l}} \\
& \cdot B\left(-\frac{s}{2}-1,-\frac{t}{2}+1\right)\left(-\frac{1}{M}\right)^{q_{1}} \\
& \cdot \sum_{j=0}^{r_{1}}\left(\begin{array}{c}
r_{1} \\
j
\end{array}\right)\left(\frac{2}{\tilde{t}^{\prime}}\right)^{j}\left(-\frac{t}{2}-1\right)_{j} U\left(-q_{1}, \frac{t}{2}+2-j-q_{1}, \frac{\tilde{t}}{2}\right) .
\end{aligned}
$$

$U$ in Eqs.(2.21) and (2.22) is the Kummer function of the second kind and is defined to be

$$
U(a, c, x)=\frac{\pi}{\sin \pi c}\left[\frac{M(a, c, x)}{(a-c) !(c-1) !}-\frac{x^{1-c} M(a+1-c, 2-c, x)}{(a-1) !(1-c) !}\right] \quad(c \neq 2,3,4 \ldots)
$$

where $M(a, c, x)=\sum_{j=0}^{\infty} \frac{(a)_{j}}{(c)_{j}} \frac{x^{j}}{j !}$ is the Kummer function of the first kind. $U$ and $M$ are the two solutions of the Kummer equation

$$
x y^{\prime \prime}(x)+(c-x) y^{\prime}(x)-a y(x)=0 .
$$

It is interesting to note that the Regge behavior is universal and is mass level independent [10]

$$
B\left(-1-\frac{s}{2},-1-\frac{t}{2}\right) \sim s^{\alpha(t)} \quad(\text { in the RR })
$$

where $\alpha(t)=\alpha(0)+\alpha^{\prime} t, \quad \alpha(0)=1$ and $\alpha^{\prime}=1 / 2$. That is, the well known $\sim s^{\alpha(t)}$ powerlaw behavior of the four tachyon string scattering amplitude in the RR can be extended to arbitrary higher string states. This result will be used to construct an inter-mass level recurrence relation for Regge string scattering amplitudes later in Eq.(3.95).

At this point, it is crucial to note that, in our case of Eq.(2.21) and Eq.(2.22), $c=c(t)$ and is not a constant as in the usual definition, so $U$ in the Regge string amplitudes is not a solution of the Kummer equation. This will make some analysis more complicated On the contrary, since $a=-q_{1}\left(\right.$ or $\left.-r_{1}\right)$ a nonpositive integer, the Kummer functions in Eq.(2.21) and Eq.(2.22) terminated to a finite sum. This will simplify the manipulation of Kummer 
functions used in this paper. For example, since $c=c(t)$ is not a constant, derivative relations of Kummer functions in Eq.(A.3) to Eq.(A.8) are no longer true. However, the recurrence relations in Eq.(A.9) to Eq.(A.14) are still valid as they can be easily verified for the case of finite number of terms. There is another form of Kummer function and is given by

$$
\begin{aligned}
U(a, c, x) & =\frac{1}{x^{a}}{ }_{2} F_{0}\left(a, 1+a-c,-\frac{1}{x}\right) \\
& \equiv \frac{1}{x^{a}} \sum_{k=0}^{\infty} \frac{1}{k !}(a)_{k}(1+a-c)_{k}\left(-\frac{1}{x}\right)^{k} .
\end{aligned}
$$

It is easy to see that the summation in Eq.(2.26) terminates to a finite sum for a nonpositive integer $a$.

\section{RECURRENCE RELATIONS AND RR STRINGY WARD IDENTITIES}

In this section, we first discuss Regge stringy Ward identities derived from Regge zeronorm states for mass level $M^{2}=2$ and 4 . We will see that, unlike the case for the GR we did in Eq.(2.7) and Eq.(2.8), Regge string Ward identities are not good enough to solve all Regge string scattering amplitudes algebraically. On the other hand, we found that the recurrence relations of Kummer functions Eq.(A.9) to Eq.(A.14) discussed in the appendix can be used to prove all Regge stringy Ward identities. Presumably the calculation can be generalized to arbitrary mass levels. Another reason to work on recurrence relations of Kummer functions instead of Regge stringy Ward identities is that the former is very easy to generalize to arbitrary higher mass levels while the latter is not.

Most importantly, for Kummer functions $U(a, c, x)$ in Regge string amplitudes in Eq.(2.21) and Eq.(2.22) with $a=-q_{1}\left(\right.$ or $\left.-r_{1}\right)$ a nonpositive integer, one can use recurrence relations to solve all $U\left(-q_{1}, c, x\right)$ functions algebraically and thus determine all Regge string scattering amplitudes at arbitrary mass levels algebraically up to multiplicative factors. We stress that for general values of $a$, the best one can obtain from recurrence relations is to express any Kummer function in terms of any two of its associated function (see the appendix).

There are 9 Regge string amplitudes for the mass level $M^{2}=2$, $T^{P P}\left(\alpha_{-1}^{P} \alpha_{-1}^{P}\right), T^{P L}\left(\alpha_{-1}^{P} \alpha_{-1}^{L}\right), T^{P T}\left(\alpha_{-1}^{P} \alpha_{-1}^{T}\right), T^{L L}\left(\alpha_{-1}^{T} \alpha_{-1}^{T}\right), \quad T^{L T}\left(\alpha_{-1}^{L} \alpha_{-1}^{T}\right), T^{T T}\left(\alpha_{-1}^{T} \alpha_{-1}^{T}\right)$, 
$T^{P}\left(\alpha_{-2}^{P}\right), T^{L}\left(\alpha_{-2}^{L}\right), T^{T}\left(\alpha_{-2}^{T}\right)$. For this mass level $\tilde{t}=t, \tilde{t}^{\prime}=t+4$. The Regge zero-norm states (RZNS) in Eq.(B.6) and Eq.(B.7) gives two Regge stringy Ward identities

$$
\begin{aligned}
& T^{T}-\sqrt{2} T^{P T}=0, \\
& T^{L}-\sqrt{2} T^{P L}=0 .
\end{aligned}
$$

The RZNS in Eq.(B.5) gives

$$
\sqrt{2} T^{P}-T^{P P}-\frac{1}{5} T^{L L}-\frac{1}{5} T^{T T}=0
$$

It's obvious to see that these three Regge stringy Ward identities Eq.(B.6) to Eq.(B.5 $)$ are not good enough to solve all the 9 Regge string scattering amplitudes algebraically. Indeed, the amplitude $T^{L T}$ does not even show up in any of these three Ward identities.

Instead of Regge stringy Ward identities, in the following we will do the calculation based on recurrence relations of Kummer functions. We want to prove these three Regge stringy Ward identities by using recurrence relations

$$
\begin{array}{r}
U(a-1, c, x)-(2 a-c+x) U(a, c, x)+a(1+a-c) U(a+1, c, x)=0, \\
U(a, c, x)-a U(a+1, c, x)-U(a, c-1, x)=0 .
\end{array}
$$

First, by taking some special values of arguments of Kummer function in Eq.(3.30) and Eq.(3.31), one easily obtain

$$
\begin{gathered}
U(-1, x, x)=0 \\
U(-2, x, x)+x U(0, x, x)=0
\end{gathered}
$$

and

$$
U(0, c, x)-U(0, c-1, x)=0 .
$$

By using Eq.(2.22), one easily see that the Ward identity Eq.(3.27) implies

$$
U\left(0, \frac{t}{2}+2, \frac{\tilde{t}}{2}\right)+U\left(-1, \frac{t}{2}+1, \frac{\tilde{t}}{2}\right)=0
$$

To prove Eq.(3.35) by recurrence relations, we note that for the case of $a=0, c=\frac{t}{2}+1, x=$ $\frac{\tilde{t}}{2}$, Eq. (3.30) says

$$
U\left(-1, \frac{t}{2}+1, \frac{\tilde{t}}{2}\right)+U\left(0, \frac{t}{2}+1, \frac{\tilde{t}}{2}\right)=0
$$


We then apply Eq.(3.34) for the second term of Eq.(3.36) to obtain Eq.(3.35). This completes the proof of Regge stringy Ward identity Eq.(3.27) based on recurrence relations Eq.(3.30) and Eq.(3.31). The Ward identity in Eq.(3.28) implies

$$
\frac{1}{\sqrt{2}} \frac{\tilde{t}^{\prime}}{2}\left[U\left(0, \frac{t}{2}+2, \frac{\tilde{t}}{2}\right)+U\left(-1, \frac{t}{2}+1, \frac{\tilde{t}}{2}\right)\right]+\left(-\frac{t}{2}-1\right) U\left(-1, \frac{t}{2}, \frac{\tilde{t}}{2}\right)=0 .
$$

To prove Eq.(3.37) by using recurrence relations, we note that Eq.(3.35) implies the first and the second terms of Eq.(3.37) cancel out. Eq.(3.34) and $t=\tilde{t}$ say that the last term of Eq.(3.37) vanishes. Finally, to prove Eq.(3.29) by using recurrence relations, one needs to prove

$$
\begin{aligned}
& {\left[\frac{1}{10}\left(\frac{\tilde{t}^{\prime}}{2}\right)^{2}+\frac{\tilde{t}}{2}-\frac{t}{5}\right] U\left(0, \frac{t}{2}+2, \frac{\tilde{t}}{2}\right)+\frac{1}{2} U\left(-2, \frac{t}{2}, \frac{\tilde{t}}{2}\right)} \\
& +\frac{1}{5}\left(\frac{\tilde{t}^{\prime}}{2}\right)\left(-\frac{t}{2}-1\right) U\left(0, \frac{t}{2}+1, \frac{\tilde{t}}{2}\right)+\frac{1}{10}\left(-\frac{t}{2}-1\right)\left(-\frac{t}{2}\right) U\left(0, \frac{t}{2}, \frac{\tilde{t}}{2}\right)=0 .
\end{aligned}
$$

Now Eq.(3.33) implies

$$
U\left(0, \frac{t}{2}+2, \frac{\tilde{t}}{2}\right)=U\left(0, \frac{t}{2}+1, \frac{\tilde{t}}{2}\right)=U\left(0, \frac{t}{2}, \frac{\tilde{t}}{2}\right) .
$$

Therefore Eq.(3.38) is equivalent to

$$
\frac{t}{2} U\left(0, \frac{t}{2}, \frac{\tilde{t}}{2}\right)+U\left(-2, \frac{t}{2}, \frac{\tilde{t}}{2}\right)=0 .
$$

Finally one can use Eq.(3.33) and $\tilde{t}=t$ to prove Eq.(3.40). This completes the proof of Regge stringy Ward identities for mass level $M^{2}=2$ by using recurrence relations of Kummer functions.

We now turn to the case of mass level $M^{2}=4$. There are 22 Regge string amplitudes for the mass level $M^{2}=4, T^{P P P}, T^{P P L}, T^{P P T}, T^{P L L}, T^{P L T}, T^{P T T}, T^{P P}, T^{L P}, T^{T P}, T^{L L L}$, $T^{L L T}, T^{L T T}, T^{T T T}, T^{P L}, T^{L L}, T^{T L}, T^{P T}, T^{L T}, T^{T T}, T^{P}, T^{L}, T^{T}$. To fix the notation, we adopt the convention of mass ordered in the $\alpha_{-n}^{\alpha}$ operators, for example, $T^{L T}\left(\alpha_{-2}^{L} \alpha_{-1}^{T}\right)$ and $T^{T L}\left(\alpha_{-2}^{T} \alpha_{-1}^{L}\right)$ etc. For this mass level $\tilde{t}=t-2, \tilde{t}^{\prime}=t+6$. The 8 RZNS Eqs.(B.12), (B.16), $(\mathrm{B} .17),(\overline{\mathrm{B} .18}),(\overline{\mathrm{B} .20}),(\overline{\mathrm{B} .21}),(\overline{\mathrm{B} .22})$ and $(\overline{\mathrm{B} .23})$ calculated in the appendix B give 8 Regge stringy Ward identities

$$
\begin{gathered}
25 T^{P P P}+9 T^{P L L}+9 T^{P T T}-9 T^{L L}-9 T^{T T}-75 T^{P P}+50 T^{P}=0, \\
T^{P L L}-T^{L L}=0,
\end{gathered}
$$




$$
\begin{gathered}
T^{P T T}-T^{T T}=0, \\
T^{P L T}-T^{(L T)}=0, \\
9 T^{P P T}+T^{L L T}+T^{T T T}-18 T^{(P T)}+6 T^{T}=0, \\
9 T^{P P L}+T^{L L L}+T^{L T T}-18 T^{(P L)}+6 T^{L}=0, \\
T^{L L T}+T^{T T T}-9 T^{[P T]}-3 T^{T}=0, \\
T^{L L L}+T^{L T T}-9 T^{[P L]}-3 T^{L}=0 .
\end{gathered}
$$

It is obvious to see that these eight Regge stringy Ward identities are not good enough to solve the 22 Regge string scattering amplitudes algebraically. Indeed, for example, the amplitude $T^{[L T]}$ does not even show up in any of these eight Ward identities. However, in the GR, one can identify $e^{P}$ and $e^{L}$ components [11,12] (Correspondingly the creation operators $\alpha_{-n}^{P}$ and $-\alpha_{-n}^{L}$ are identified, where the sign comes from the difference between the timelike and spacelike directions specified by the metric of the scattering plane $\eta_{\mu \nu}=\operatorname{diag}(-1,1,1)$ .), and take high-energy fixed angle limit to get three Ward identities in leading order energy $[11,12]$

$$
\begin{aligned}
T^{L L T}+T^{(L T)} & =0, \\
10 T^{L L T}+T^{T T T}+18 T^{(L T)} & =0, \\
T^{L L T}+T^{T T T}+9 T^{[L T]} & =0,
\end{aligned}
$$

which can be easily solved to get [11, 12]

$$
T^{T T T}: T^{L L T}: T^{(L T)}: T^{[L T]}=8: 1:-1:-1 .
$$

The ratios above are consistent with Eq.(2.4).

For illustration, we now proceed to prove Regge stringy Ward identities Eq.(3.41) to Eq.(3.44) by using recurrence relations Eq.(3.30), Eq.(3.31) and

$$
(c-a-1) U(a, c-1, x)-(x+c-1) U(a, c, x)+x U(a, c+1, x)=0 .
$$

Other Regge stringy Ward identities Eq.(3.45) to Eq.(3.48) can be similarly proved by using recurrence relations. For the case of $a=-1, c=x+1$, Eq.(3.53) reduces to 


$$
(x+1) U(-1, x, x)-2 x U(-1, x+1, x)+x U(-1, x+2, x)=0 .
$$

For the case of $a=-1, c=x+2$, Eq.(3.31) reduces to

$$
U(-1, x+2, x)+U(0, x+2, x)-U(-1, x+1, x)=0 .
$$

Finally Eq.(3.54), Eq.(3.55), and Eq.(3.32) say

$$
\begin{gathered}
U(-1, x+2, x)=-2 U(0, x+2, x), \\
U(-1, x+1, x)=-U(0, x+2, x) .
\end{gathered}
$$

We are now ready to prove Regge stringy Ward identities. We first prove Regge stringy Ward identity Eq.(3.42). The two terms in Eq.(3.42) divided by the beta function can be calculated to be

$$
\begin{aligned}
\frac{1}{B} T^{P L L}= & -\frac{1}{M}\left(\frac{\tilde{t}^{\prime}}{2 M}\right)^{2}\left[U\left(-1, \frac{t}{2}+1, \frac{t}{2}-1\right)+2\left(\frac{2}{\tilde{t}^{\prime}}\right)\left(-\frac{t}{2}-1\right) U\left(-1, \frac{t}{2}, \frac{t}{2}-1\right)\right. \\
& \left.+\left(\frac{2}{\tilde{t}^{\prime}}\right)^{2}\left(-\frac{t}{2}-1\right)\left(-\frac{t}{2}\right) U\left(-1, \frac{t}{2}-1, \frac{t}{2}-1\right)\right] \\
= & -\frac{1}{M}\left(\frac{t+6}{2 M}\right)^{2}\left[\begin{array}{c}
\left.U\left(-1, \frac{t}{2}+1, \frac{t}{2}-1\right)-2 \frac{t+2}{t+6} U\left(-1, \frac{t}{2}, \frac{t}{2}-1\right)\right] \\
+\frac{t(t+2)}{(t+6)^{2}} U\left(-1, \frac{t}{2}-1, \frac{t}{2}-1\right)
\end{array}\right] \\
\frac{1}{B} T^{L L}= & \left(\frac{\tilde{t}^{\prime}}{2 M}\right)^{2}\left[U\left(0, \frac{t}{2}+2, \frac{t}{2}-1\right)+\left(\frac{2}{\tilde{t}^{\prime}}\right)\left(-\frac{t}{2}-1\right) U\left(0, \frac{t}{2}+1, \frac{t}{2}-1\right)\right] \\
= & \left(\frac{t+6}{2 M}\right)^{2}\left[U\left(0, \frac{t}{2}+2, \frac{t}{2}-1\right)-\frac{t+2}{t+6} U\left(0, \frac{t}{2}+1, \frac{t}{2}-1\right)\right] .
\end{aligned}
$$

Therefore we want to show

$$
\begin{gathered}
-\frac{1}{M}\left[U\left(-1, \frac{t}{2}+1, \frac{t}{2}-1\right)-2 \frac{t+2}{t+6} U\left(-1, \frac{t}{2}, \frac{t}{2}-1\right)+\frac{t(t+2)}{(t+6)^{2}} U\left(-1, \frac{t}{2}-1, \frac{t}{2}-1\right)\right] \\
-U\left(0, \frac{t}{2}+2, \frac{t}{2}-1\right)+\frac{t+2}{t+6} U\left(0, \frac{t}{2}+1, \frac{t}{2}-1\right) \stackrel{?}{=} 0
\end{gathered}
$$

Eq.(3.32) implies the third term of Eq.(3.60) vanishes and therefore Eq.(3.34) implies that Eq.(3.60) is equivalent to

$$
\begin{aligned}
& -\frac{1}{M} U\left(-1, \frac{t}{2}+1, \frac{t}{2}-1\right)+\frac{2}{M} \frac{t+2}{t+6} U\left(-1, \frac{t}{2}, \frac{t}{2}-1\right)-\frac{4}{t+6} U\left(0, \frac{t}{2}+1, \frac{t}{2}-1\right) \\
& =\frac{1}{M}\left[-U\left(-1, \frac{t}{2}+1, \frac{t}{2}-1\right)+2 \frac{t+2}{t+6} U\left(-1, \frac{t}{2}, \frac{t}{2}-1\right)-\frac{8}{t+6} U\left(0, \frac{t}{2}+1, \frac{t}{2}-1\right)\right] \\
& =0
\end{aligned}
$$


For the case of $x=\frac{t}{2}-1, \mathrm{Eq} \cdot(3.56)$ and Eq.(3.57) implies

$$
U\left(-1, \frac{t}{2}+1, \frac{t}{2}-1\right)=2 U\left(-1, \frac{t}{2}, \frac{t}{2}-1\right)=-2 U\left(0, \frac{t}{2}+1, \frac{t}{2}-1\right) .
$$

Hence Eq.(3.61) is easily proved.

We now prove Regge stringy Ward identity Eq.(3.43). The two terms in Eq.(3.43) divided by the beta function can be calculated to be

$$
\begin{gathered}
\frac{1}{B} T^{P T T}=(-t)\left(-\frac{1}{M}\right) U\left(-1, \frac{t}{2}+1, \frac{t}{2}-1\right), \\
\frac{1}{B} T^{T T}=(-t) U\left(0, \frac{t}{2}+2, \frac{t}{2}-1\right) .
\end{gathered}
$$

Therefore we want to show

$$
\frac{t}{M} U\left(-1, \frac{t}{2}+1, \frac{t}{2}-1\right)+t U\left(0, \frac{t}{2}+2, \frac{t}{2}-1\right) \stackrel{?}{=} 0
$$

For the case of $x=\frac{t}{2}-1$, Eq.(3.56) means

$$
U\left(-1, \frac{t}{2}+1, \frac{t}{2}-1\right)=-2 U\left(0, \frac{t}{2}+1, \frac{t}{2}-1\right) .
$$

Eq.(3.64) and Eq.(3.34) prove Eq.(3.63).

We can now turn to prove Regge stringy Ward identity Eq.(3.41). We first note that Eq.(3.42) and Eq.(3.43) implies that Eq.(3.41) is equivalent to

$$
25 T^{P P P}-75 T^{P P}+50 T^{P}=0 .
$$

The three terms in Eq.(3.65) divided by the beta function are

$$
\begin{aligned}
\frac{1}{B} T^{P} & =\left(-\frac{t-2}{2 M}\right) U\left(0, \frac{t}{2}+2, \frac{t}{2}-1\right), \\
\frac{1}{B} T^{P P} & =-\frac{1}{M}\left(-\frac{t-2}{2 M}\right) U\left(-1, \frac{t}{2}+1, \frac{t}{2}-1\right), \\
\frac{1}{B} T^{P P P} & =\left(-\frac{1}{M}\right)^{3} U\left(-3, \frac{t}{2}-1, \frac{t}{2}-1\right) .
\end{aligned}
$$

Therefore we want to show

$$
\begin{aligned}
& 2\left(-\frac{t-2}{2 M}\right) U\left(0, \frac{t}{2}+2, \frac{t}{2}-1\right)-3\left(-\frac{1}{M}\right)\left(-\frac{t-2}{2 M}\right) U\left(-1, \frac{t}{2}+1, \frac{t}{2}-1\right) \\
& +\left(-\frac{1}{M}\right)^{3} U\left(-3, \frac{t}{2}-1, \frac{t}{2}-1\right) \stackrel{?}{=} 0
\end{aligned}
$$


For the case of $a=-2, c=x$, Eq.(3.30) gives

$$
U(-3, x, x)+4 U(-2, x, x)+2(1+x) U(-1, x, x)=0 .
$$

Using Eq.(3.32), we obtain

$$
U(-3, x, x)+4 U(-2, x, x)=0 .
$$

From Eq.(3.71) and Eq.(3.33), we obtain

$$
U(-3, x, x)-4 x U(0, x, x)=0
$$

From Eq.(3.72) and Eq.(3.34), we obtain

$$
\begin{aligned}
& 2\left(-\frac{t-2}{2 M}\right) U\left(0, \frac{t}{2}+2, \frac{t}{2}-1\right)-3\left(-\frac{1}{M}\right)\left(-\frac{t-2}{2 M}\right) U\left(-1, \frac{t}{2}+1, \frac{t}{2}-1\right) \\
& +\left(-\frac{1}{M}\right)^{3} U\left(-3, \frac{t}{2}-1, \frac{t}{2}-1\right) \\
= & \left(2\left(-\frac{t-2}{2 M}\right)+4\left(\frac{t-2}{2}\right)\left(-\frac{1}{M}\right)^{3}\right) U\left(0, \frac{t}{2}+2, \frac{t}{2}-1\right) \\
& +3 \frac{1}{M}\left(-\frac{t-2}{2 M}\right) U\left(-1, \frac{t}{2}+1, \frac{t}{2}-1\right) \\
= & \left(2\left(-\frac{t-2}{4}\right)+\left(\frac{t-2}{2}\right)\left(-\frac{1}{2}\right)\right) U\left(0, \frac{t}{2}+2, \frac{t}{2}-1\right) \\
& +\frac{3}{2}\left(-\frac{t-2}{4}\right) U\left(-1, \frac{t}{2}+1, \frac{t}{2}-1\right) \\
= & \frac{t}{2}\left[-\frac{3}{2} U\left(0, \frac{t}{2}+2, \frac{t}{2}-1\right)-\frac{3}{2} \frac{1}{2} U\left(-1, \frac{t}{2}+1, \frac{t}{2}-1\right)\right] .
\end{aligned}
$$

Finally Eq.(3.34) and Eq.(3.56) implies that Eq.(3.73) vanishes. This proves Eq.(3.69).

For the fourth stringy Ward identity at mass level $M^{2}=4$, the two terms in Eq.(3.44) 
divided by the beta function are

$$
\begin{aligned}
\frac{1}{B} T^{P L T} & =-\frac{\sqrt{-t} \tilde{t}^{\prime}}{2 M^{2}}\left[U\left(-1, \frac{t}{2}+1, \frac{\tilde{t}}{2}\right)+\left(\frac{2}{\tilde{t}^{\prime}}\right)\left(-\frac{t}{2}-1\right) U\left(-1, \frac{t}{2}, \frac{\tilde{t}}{2}\right)\right] \\
& =-\frac{\sqrt{-t}(t+6)}{2 M^{2}}\left[\begin{array}{c}
\left.+\left(\frac{2}{t+6}\right)\left(-\frac{t}{2}-1\right) U\left(-1, \frac{t}{2}, \frac{t}{2}-1\right)\right] \\
\frac{1}{B} T^{L T}
\end{array}=\sqrt{-t} \frac{\tilde{t}^{\prime}}{2 M} U\left(0, \frac{t}{2}+2, \frac{\tilde{t}}{2}\right)=\sqrt{-t} \frac{t+6}{2 M} U\left(0, \frac{t}{2}+2, \frac{t}{2}-1\right)\right. \\
\frac{1}{B} T^{T L} & =\sqrt{-t} \frac{\tilde{t}^{\prime}}{2 M}\left[U\left(0, \frac{t}{2}+2, \frac{\tilde{t}}{2}\right)+\left(\frac{2}{\tilde{t^{\prime}}}\right)\left(-\frac{t}{2}-1\right) U\left(0, \frac{t}{2}+1, \frac{\tilde{t}}{2}\right)\right] \\
& =\sqrt{-t} \frac{t+6}{2 M}\left[U\left(0, \frac{t}{2}+2, \frac{t}{2}-1\right)+\left(\frac{2}{t+6}\right)\left(-\frac{t}{2}-1\right) U\left(0, \frac{t}{2}+1, \frac{t}{2}-1\right)\right] .
\end{aligned}
$$

Therefore we want to show

$$
\begin{aligned}
& 2 T^{P L T}-T^{L T}-T^{T L} \\
&= B \sqrt{t}\left[-\frac{t+6}{M^{2}} U\left(-1, \frac{t}{2}+1, \frac{t}{2}-1\right)-\frac{2}{M^{2}}\left(-\frac{t}{2}-1\right) U\left(-1, \frac{t}{2}, \frac{t}{2}-1\right)\right. \\
&\left.-\frac{t+6}{M} U\left(0, \frac{t}{2}+2, \frac{t}{2}-1\right)-\frac{1}{M}\left(-\frac{t}{2}-1\right) U\left(0, \frac{t}{2}+1, \frac{t}{2}-1\right)\right] \stackrel{?}{=} 0 .
\end{aligned}
$$

Using Eq.(3.34), we obtain

$$
\begin{aligned}
& \frac{1}{B}\left(2 T^{P L T}-T^{L T}-T^{T L}\right) \\
& =\sqrt{t}\left[-\frac{t+6}{M^{2}} U\left(-1, \frac{t}{2}+1, \frac{t}{2}-1\right)-\frac{2}{M^{2}}\left(-\frac{t}{2}-1\right) U\left(-1, \frac{t}{2}, \frac{t}{2}-1\right)\right. \\
& \left.\quad+\frac{1}{M}\left(-t-6+\frac{t}{2}+1\right) U\left(0, \frac{t}{2}, \frac{t}{2}-1\right)\right] \\
& =\sqrt{t}\left[\begin{array}{c}
\left.-\frac{t+6}{M^{2}} U\left(-1, \frac{t}{2}+1, \frac{t}{2}-1\right)-\frac{2}{M^{2}}\left(-\frac{t}{2}-1\right) U\left(-1, \frac{t}{2}, \frac{t}{2}-1\right)\right] \\
+\frac{1}{M}\left(-\frac{t}{2}-5\right) U\left(0, \frac{t}{2}, \frac{t}{2}-1\right)
\end{array}\right] \\
& =\sqrt{t}\left[\begin{array}{c}
\left.-\frac{t+6}{4} U\left(-1, \frac{t}{2}+1, \frac{t}{2}-1\right)+\frac{t+2}{4} U\left(-1, \frac{t}{2}, \frac{t}{2}-1\right)\right] \\
-\frac{t+10}{4} U\left(0, \frac{t}{2}, \frac{t}{2}-1\right)
\end{array}\right]
\end{aligned}
$$

One can now use Eq.(3.56) and Eq.(3.57) to prove that Eq.(3.78) vanishes. This completes the explicit proof of four Regge stringy Ward identities for mass level $M^{2}=4$ by using recurrence relations of Kummer functions. Other four Regge stringy Ward identities can be similarly proved. 
We observe that the recurrence relations of Kummer functions are more powerful than Regge stringy Ward identities in relating Regge string scattering amplitudes. This is indeed the case as we will show now in the following that all Regge string scattering amplitudes can be algebraically solved by using recurrence relations up to multiplicative factors in the first line of Eq.(2.21) (Eq.(2.22) ).

To be more precise, we will first show that the ratio

$$
\frac{U(a, c, x)}{U(0, x, x)}=f(a, c, x), a=0,-1,-2,-3, \ldots
$$

is fixed and can be calculated by using recurrence relations Eq.(3.30), Eq.(3.31) and

$$
(c-a) U(a, c, x)+U(a-1, c, x)-x U(a, c+1, x)=0 .
$$

We stress that Eq.(3.79) is nontrivial in the sense that, for general values of $a$, the best one can obtain from recurrence relations is to express any Kummer function in terms of any two of its associated function (see Appendix A). However, Eq.(3.79) states that for nonpositive integer values of $a, U(a, c, x)$ can be fixed up to an overall factor by using recurrence relations.

To prove Eq.(3.79), we first note that, for $a=0, c=x$, recurrence relation Eq.(3.30) implies Eq.(3.32). This determines $\frac{U(a, x, x)}{U(0, x, x)}$ for $a$ is a nonpositive integer. For illustration, we list examples of relations

$$
\begin{aligned}
& a=-1, U(-2, x, x)+0+x U(0, x, x)=0, \\
& a=-2, U(-3, x, x)+4 U(-2, x, x)+0=0, \\
& a=-3, U(-4, x, x)+6 U(-3, x, x)+3(2+x) U(-2, x, x)=0,
\end{aligned}
$$

which determines $\frac{U(-2, x, x)}{U(0, x, x)}, \frac{U(-3, x, x)}{U(0, x, x)}, \frac{U(-4, x, x)}{U(0, x, x)}, \ldots$ recursively.

Next we extend the result to $\frac{U(a, c, x)}{U(0, x, x)}$ for $c=x+Z, Z=$ integer. We first consider the simple case with $a=0$. From Eq.(3.31), we obtain for $a=0, c=x+i, i \in Z$

$$
U(0, x+i, x)-U(0, x+i-1, x)=0,
$$

which gives $\frac{U(0, x+i, x)}{U(0, x, x)}=1$. This proves Eq.(3.79) for $a=0$. For $a \in Z_{-}, c=x+Z_{-}$, we obtain from Eq.(3.31) with $c=x-i$

$$
U(a, x-i, x)-a U(a+1, x-i, x)-U(a, x-i-1, x)=0 .
$$


Since $\frac{U(a, x, x)}{U(0, x, x)}, \frac{U(a+1, x, x)}{U(0, x, x)}$ have been determined for $a \in Z_{-}$, this determines $\frac{U(a, x-i, x)}{U(0, x, x)}$ for $a \in$ $Z_{-}, i=1,2,3 \ldots$ recursively. For $a \in Z_{-}, c=x+Z_{+}$, we obtain from Eq.(3.80) with $c=x+i$

$$
(x-a+i) U(a, x+i, x)+U(a-1, x+i, x)-x U(a, x+i+1, x)=0 .
$$

Since $\frac{U(a-1, x, x)}{U(0, x, x)}, \frac{U(a, x, x)}{U(0, x, x)}$ have been determined for $a \in Z_{-}$, this determines $\frac{U(a, x+i, x)}{U(0, x, x)}$ for $a \in$ $Z_{-}, i=1,2,3 \ldots$ recursively. This completes the proof of Eq.(3.79) by using recurrence relations of Kummer functions.

Secondly, we want to show that each Kummer function in the summation of Eq.(2.22) can be expressed in terms of Regge string scattering amplitudes. To show this, we first consider $r_{1}=0$ amplitudes in a fixed mass level and a fixed $q_{1}$ with no summation over Kummer functions. These amplitudes contain only one Kummer function. Then let us take the amplitude with the maximum $p_{1}$. By decreasing $p_{1}$ and increasing $r_{1}$ by 1 , we can create an amplitude with two Kummer functions in the same mass level and the same $q_{1}$. The first one of the two Kummer functions is the one appeared in the previous amplitude with $r_{1}=0$, so we can write the second Kummer function in terms of the two amplitudes, one with $r_{1}=0$ and the other with $r_{1}=1$. By decreasing $p_{1}$ and increasing $r_{1}$ by 1 again, we can create an amplitude with three Kummer functions in the same mass level and the same $q_{1}$. The first two of the three Kummer functions is the ones appeared in the previous two amplitudes, so we can write the third Kummer functions in terms of the three amplitudes. We can repeat this process until $p_{1}=0$. In this way, we can express all the Kummer functions in Eq.(2.22) in terms of the RR amplitudes.

In the following, as an example, let us illustrate the above process for the mass level 4 amplitudes. There are 22 Regge string amplitudes for the mass level $M^{2}=4$. We first consider the group of amplitudes with $q_{1}=0,\left(T^{T T T}, T^{L T T}, T^{L L T}, T^{L L L}\right)$. The corresponding $r_{1}$ for each amplitude are $(0,1,2,3)$. By using Eq.(2.22), one can easily see that $U\left(0, \frac{t}{2}+2, \frac{t}{2}-1\right)$ can be expressed in terms of $T^{T T T}, U\left(0, \frac{t}{2}+1, \frac{t}{2}-1\right)$ can be expressed in terms of $\left(T^{T T T}, T^{L T T}\right), U\left(0, \frac{t}{2}, \frac{t}{2}-1\right)$ can be expressed in terms of $\left(T^{T T T}, T^{L T T}, T^{L L T}\right)$, and finally $U\left(0, \frac{t}{2}-1, \frac{t}{2}-1\right)$ can be expressed in terms of $\left(T^{T T T}, T^{L T T}, T^{L L T}, T^{L L L}\right)$. Similarly, we can consider groups of amplitudes $\left(T^{P T}, T^{P L}\right),\left(T^{L T}, T^{L L}\right)$ and $\left(T^{T T}, T^{T L}\right)$ with $q_{1}=0$; group of amplitude $\left(T^{P T T}, T^{P L T}, T^{P L L}\right)$ with $q_{1}=1$ and group of amplitude $\left(T^{P P T}, T^{P P L}\right)$ with $q_{1}=2$. All the remaining 7 amplitudes are with $r_{1}=0$, and each amplitude contains only one Kummer function. Due to the multiplicative factors, there are much more RR 
amplitudes than the number of Kummer functions involved at each fixed mass level. At mass level 4, for example, there are 22 RR amplitudes and only 10 Kummer functions involved. So there is an onto correspondence between RR amplitudes and Kummer functions. We have done the analysis by using Eq.(2.22). Similar analysis can be performed by using Eq.(2.21) to get the same results.

An important application of the above prescription is the construction of an infinite number of recurrence relations among Regge string scattering amplitudes. One can use the recurrence relations of Kummer functions Eq.(A.9) to Eq.(A.14) to systematically construct recurrence relations among Regge string scattering amplitudes.

Note that a simple calculation by using the explicit form of Kummer function in Eq.(2.26) gives $U(0, x, x)=1$. However, when applying to the case of Regge string scattering amplitudes, it will bring back a multiplicative factor in the first line of Eq.(2.21), (Eq.(2.22)) for each amplitude. We thus conclude that all Regge string scattering amplitudes can be algebraically solved by recurrence relations of Kummer functions up to multiplicative factors.

Finally we calculate some examples of recurrence relations among Regge string scattering amplitudes. At mass level $M^{2}=2$, by using Eq.(2.22) and the recurrence relation

$$
U\left(-2, \frac{t}{2}, \frac{t}{2}\right)+\left(\frac{t}{2}+1\right) U\left(-1, \frac{t}{2}, \frac{t}{2}\right)-\frac{t}{2} U\left(-1, \frac{t}{2}+1, \frac{t}{2}\right)=0
$$

one can obtain the following recurrence relation among Regge string scattering amplitudes

$$
M \sqrt{-t} T^{P P}-\frac{t}{2} T^{P T}=0
$$

In contrast to the Regge stringy Ward identities Eq.(3.27), Eq.(3.28) and Eq.(3.29) which contain only constant coefficients, the recurrence relation in Eq.(3.85) contains kinematic variable $t$ in its coefficients. Note that Eq.(3.85) is independent of all three Regge stringy Ward identities at mass level $M^{2}=2$.

At mass level $M^{2}=4$, by using Eq.(2.22), one can calculate

$$
\begin{aligned}
\frac{1}{B} T^{P P P} & =\left(-\frac{1}{M}\right)^{3} U\left(-3, \frac{t}{2}-1, \frac{t}{2}-1\right), \\
\frac{1}{B} T^{P P T} & =\left(-\frac{1}{M}\right)^{2} \sqrt{-t} U\left(-2, \frac{t}{2}, \frac{t}{2}-1\right), \\
\frac{1}{B} T^{P P L} & =\frac{t+6}{2 M^{3}} U\left(-2, \frac{t}{2}, \frac{t}{2}-1\right)+\frac{1}{M^{3}}\left(-\frac{t}{2}-1\right) U\left(-2, \frac{t}{2}-1, \frac{t}{2}-1\right) .
\end{aligned}
$$


The recurrence relation

$$
U\left(-3, \frac{t}{2}-1, \frac{t}{2}-1\right)+\left(\frac{t}{2}+1\right) U\left(-2, \frac{t}{2}-1, \frac{t}{2}-1\right)-\left(\frac{t}{2}-1\right) U\left(-2, \frac{t}{2}, \frac{t}{2}-1\right)=0
$$

leads to the following recurrence relation among Regge string scattering amplitudes

$$
M \sqrt{-t} T^{P P P}-4 T^{P P T}+M \sqrt{-t} T^{P P L}=0 .
$$

We have explicitly verified Eq.(3.85) and Eq.(3.90). It will be difficult to identify identity like Eq.(3.90) without using the recurrence relation Eq.(3.89). One can similarly construct infinite number of them for amplitudes at arbitrary higher mass levels based on the recurrence relations of Kummer functions and their associated functions (see Appendix A).

The recurrence relations Eq.(3.85) and Eq.(3.90) for Regge string scattering amplitudes are reminiscent of four point BCJ relations [27-31] for Yang-Mills gluon color-stripped scattering amplitudes $A$

$$
\begin{aligned}
& t A\left(k_{1}, k_{4}, k_{2}, k_{3}\right)-s A\left(k_{1}, k_{3}, k_{4}, k_{2}\right)=0, \\
& s A\left(k_{1}, k_{2}, k_{3}, k_{4}\right)-u A\left(k_{1}, k_{4}, k_{2}, k_{3}\right)=0, \\
& u A\left(k_{1}, k_{3}, k_{4}, k_{2}\right)-t A\left(k_{1}, k_{2}, k_{3}, k_{4}\right)=0
\end{aligned}
$$

where non-constant coefficients, or kinematic variables, show up in the relations. Note that other relation such as four point KK relation [32, 33]

$$
A\left(k_{1}, k_{2}, k_{3}, k_{4}\right)+A\left(k_{1}, k_{3}, k_{4}, k_{2}\right)+A\left(k_{1}, k_{4}, k_{2}, k_{3}\right)=0
$$

contains only constant coefficients which is similar to Regge stringy Ward identities.

For the third example, we construct an inter-mass level recurrence relation for Regge string scattering amplitudes at mass level $M^{2}=2,4$. We begin with the addition theorem of Kummer function [26]

$$
U(a, c, x+y)=\sum_{k=0}^{\infty} \frac{1}{k !}(a)_{k}(-1)^{k} y^{k} U(a+k, c+k, x)
$$

which terminates to a finite sum for a nonpositive integer $a$. By taking, for example, $a=$ $-1, c=\frac{t}{2}+1, x=\frac{t}{2}-1$ and $y=1$, the theorem gives

$$
U\left(-1, \frac{t}{2}+1, \frac{t}{2}\right)-U\left(-1, \frac{t}{2}+1, \frac{t}{2}-1\right)-U\left(0, \frac{t}{2}+2, \frac{t}{2}-1\right)=0 .
$$


Note that, unlike all previous cases, the last arguments of Kummer functions in Eq.(3.94) can be different. Eq.(3.94) leads to an inter-mass level recurrence relation

$$
M(2)(t+6) T_{2}^{T P}-2 M(4)^{2} \sqrt{-t} T_{4}^{L P}+2 M(4) T_{4}^{L T}=0
$$

where masses $M(2)=\sqrt{2}, M(4)=\sqrt{4}=2$, and $T_{2}, T_{4}$ are Regge string scattering am-

plitudes for mass levels $M^{2}=2,4$ respectively. In deriving Eq.(3.95), it is important to use the fact that the Regge power law behavior in Eq.(2.25) is universal and is mass level independent [10].

Following the same procedure, one can construct infinite number of recurrence relations among Regge string scattering amplitudes at arbitrary mass levels which, in general, are independent of Regge stringy Ward identities. The physical origin for the four point BCJ relations Eq.(3.91), for example, can be traced back to the conservation of momenta. On the contrary, the physical origin of these new recurrence relations among Regge string scattering amplitudes or "symmetries" is not well understood and is an interesting problem to study.

\section{CONCLUSION}

In this paper, we calculate the complete set of high-energy string scattering amplitudes in the Regge regime. We derive Regge stringy Ward identities for the first few mass levels based on the decoupling of zero-norm states. These results are valid even for higher point functions and higher point loops by unitarity. We found that, unlike the case for the fixed angle regime, the Regge stringy Ward identities were not good enough to solve all the Regge string scattering amplitudes algebraically. On the other hand, we found that all the Regge stringy Ward identities can be explicitly proved by the recurrence relations of Kummer functions of the second kind. We then show that, instead of Regge stringy Ward identities, one can use these recurrence relations to solve all Regge string scattering amplitudes algebraically up to multiplicative factors.

Finally, for illustration, we calculate some examples of recurrence relations among Regge string scattering amplitudes of different string states based on recurrence relations and addition theorem of Kummer functions. In contrast to the Regge stringy Ward identities which contain only constant coefficients, these recurrence relations contains kinematic variable $t$ in its coefficients and are in general independent of Regge stringy Ward identities. The dy- 
namical origin of these recurrence relations remain to be studied. These recurrence relations among Regge string scattering amplitudes are dual to linear relations or symmetries among high-energy fixed angle string scattering amplitudes discovered previously [11 14].

Recently, five-point tachyon amplitude was considered in the context of BCFW application of string theory in [34]. It will be interesting to consider both RR and GR of higher spin five-point scattering amplitudes.

\section{ACKNOWLEDGMENTS}

We thank Yung-Yeh Chang, Chih-Hao Fu, Song He, Yu-Ting Huang, Chung-I Tan and Yi Yang for helpful discussions. This work is supported in part by the National Science Council, 50 billions project of Ministry of Education, National Center for Theoretical Sciences and S.T. Yau center of NCTU, Taiwan.

\section{Appendix A: Recurrence Relations of Kummer Functions}

In this appendix, we review the recurrence relations of Kummer functions of the second kind [26]. The Kummer function of the second kind $U$ is defined to be

$$
U(a, c, x)=\frac{\pi}{\sin \pi c}\left[\frac{M(a, c, x)}{(a-c) !(c-1) !}-\frac{x^{1-c} M(a+1-c, 2-c, x)}{(a-1) !(1-c) !}\right] \quad(c \neq 2,3,4 \ldots)
$$

where $M(a, c, x)=\sum_{j=0}^{\infty} \frac{(a)_{j}}{(c)_{j}} \frac{x^{j}}{j !}$ is the Kummer function of the first kind. Here $(a)_{j}=$ $a(a+1)(a+2) \ldots(a+j-1)$ is the Pochhammer symbol. $U$ and $M$ are the two solutions of the Kummer Equation

$$
x y^{\prime \prime}(x)+(c-x) y^{\prime}(x)-a y(x)=0 .
$$

For any confluent hypergeometric function with parameters $(a, c)$ the four functions with parameters $(a-1, c),(a+1, c),(a, c-1)$ and $(a, c+1)$ are called the contiguous functions. 
It follows, from the Kummer Equation Eq.(A.2) and derivatives of Kummer functions

$$
\begin{aligned}
U(a+1, c+1, x) & =\frac{-1}{a} U^{\prime}(a, c, x), \\
U(a+1, c, x) & =\frac{1}{1+a-c} U(a, c, x)+\frac{x}{a(1+a-c)} U^{\prime}(a, c, x), \\
U(a, c-1, x) & =\frac{1-c}{1+a-c} U(a, c, x)-\frac{x}{1+a-c} U^{\prime}(a, c, x), \\
U(a, c+1, x) & =U^{\prime}(a, c, x)-U^{\prime}(a, c, x), \\
U(a-1, c, x) & =(x+a-c) U(a, c, x)-x U^{\prime}(a, c, x), \\
U(a-1, c-1, x) & =(1+x-c) U(a, c, x)-x U^{\prime}(a, c, x),
\end{aligned}
$$

that a recurrence relation exists between any such function and any two of its contiguous functions. There are six recurrence relations

$$
\begin{aligned}
U(a-1, c, x)-(2 a-c+x) U(a, c, x)+a(1+a-c) U(a+1, c, x) & =0 \\
(c-a-1) U(a, c-1, x)-(x+c-1)) U(a, c, x)+x U(a, c+1, x) & =0 \\
U(a, c, x)-a U(a+1, c, x)-U(a, c-1, x) & =0 \\
(c-a) U(a, c, x)+U(a-1, c, x)-x U(a, c+1, x) & =0 \\
(a+x) U(a, c, x)-x U(a, c+1, x)+a(c-a-1) U(a+1, c, x) & =0 \\
(a+x-1) U(a, c, x)-U(a-1, c, x)+(1+a-c) U(a, c-1, x) & =0 .
\end{aligned}
$$

From any two of these six relations the remaining four recurrence relations can be deduced. Thus they are not independent. For example, one can deduces recurrence relation Eq. (A.9) from Eq.(A.11) and Eq.(A.12). We start with Eq.(A.11) with $c \rightarrow c+1$

$$
U(a, c+1, x)-a U(a+1, c+1, x)-U(a, c, x)=0 .
$$

We consider Eq. (A.12) $+x \cdot$ Eq. (A.15) to deduce

$$
(c-a-x) U(a, c, x)+U(a-1, c, x)-a x U(a+1, c+1, x)=0 .
$$

Next we replace Eq.(A.12) with $a \rightarrow a+1$ to get

$$
(c-a-1) U(a+1, c, x)+U(a, c, x)-x U(a+1, c+1, x)=0 .
$$

Finally we consider Eq.(A.16) $-a \cdot$ Eq. (A.17) to deduce

$$
(c-2 a-x) U(a, c, x)+U(a-1, c, x)-a(c-a-1) U(a+1, c, x)=0,
$$


which is nothing but Eq.(A.9).

The confluent hypergeometric function with parameters $(a \pm m, c \pm n)$ for $m, n=$ $0,1,2 \ldots$ are called associated functions. Again it can be shown that there exist relations between any three associated functions, so that any confluent hypergeometric function can be expressed in terms of any two of its associated functions.

\section{Appendix B: Regge String Zero-Norm States}

There are two types of zero-norm states (ZNS) in the old covariant first quantized string spectrum

$$
\text { Type I : } L_{-1}|x\rangle \text {, where } L_{1}|x\rangle=L_{2}|x\rangle=0, L_{0}|x\rangle=0 \text {; }
$$

$$
\text { Type II : }\left(L_{-2}+\frac{3}{2} L_{-1}^{2}\right)|\widetilde{x}\rangle \text {, where } L_{1}|\widetilde{x}\rangle=L_{2}|\widetilde{x}\rangle=0,\left(L_{0}+1\right)|\widetilde{x}\rangle=0 \text {. }
$$

Eq. (B.1) and Eq. (B.2) can be derived from Kac determinant in conformal field theory. While type I states have zero-norm at any spacetime dimension, type II states have zero-

norm only at $\mathrm{D}=26$. The existence of type II zero-norm states signals the importance of zero-norm states in the structure of the theory of string. In fact, by requiring the decoupling of these two types of zero-norm states or stringy Ward identities in the high-energy fixed angle regime, one can calculate algebraically the complete ratios among high-energy string scattering amplitudes of different string states at each fixed mass level in Eq.(2.4).

In the RR, however, the Regge stringy Ward identities turn out to be not good enough to solve all the Regge scattering amplitudes algebraically. This is due to the much more numerous Regge string scattering amplitudes than those in the GR at each fixed mass level. In this appendix, we list all ZNS for $M^{2}=2$ and 4 and calculate their Regge limit which we use in the text to demonstrate the calculation. At the first massive level $k^{2}=-2$, there is a type II ZNS

$$
\left[\frac{1}{2} \alpha_{-1} \cdot \alpha_{-1}+\frac{5}{2} k \cdot \alpha_{-2}+\frac{3}{2}\left(k \cdot \alpha_{-1}\right)^{2}\right]|0, k\rangle
$$

and a type I ZNS 


$$
\left[\theta \cdot \alpha_{-2}+\left(k \cdot \alpha_{-1}\right)\left(\theta \cdot \alpha_{-1}\right)\right]|0, k\rangle, \theta \cdot k=0 .
$$

In the Regge limit, the polarizations of the 2nd particle with momentum $k_{2}$ on the scattering plane used in the text were defined to be $e^{P}=\frac{1}{M_{2}}\left(E_{2}, \mathrm{k}_{2}, 0\right)=\frac{k_{2}}{M_{2}}$ as the momentum polarization, $e^{L}=\frac{1}{M_{2}}\left(\mathrm{k}_{2}, E_{2}, 0\right)$ the longitudinal polarization and $e^{T}=(0,0,1)$ the transverse polarization which lies on the scattering plane. $\eta_{\mu \nu}=\operatorname{diag}(-1,1,1)$. The three vectors $e^{P}$, $e^{L}$ and $e^{T}$ satisfy the completeness relation $\eta_{\mu \nu}=\sum_{\alpha, \beta} e_{\mu}^{\alpha} e_{\nu}^{\beta} \eta_{\alpha \beta}$ where $\mu, \nu=0,1,2$ and $\alpha, \beta=P, L, T$ and $\alpha_{-1}^{T}=\sum_{\mu} e_{\mu}^{T} \alpha_{-1}^{\mu}, \alpha_{-1}^{T} \alpha_{-2}^{L}=\sum_{\mu, \nu} e_{\mu}^{T} e_{\nu}^{L} \alpha_{-1}^{\mu} \alpha_{-2}^{\nu}$ etc.

In the Regge limit, the type II ZNS in Eq.(B.3) gives the Regge zero-norm state (RZNS)

$$
\left(\sqrt{2} \alpha_{-2}^{P}-\alpha_{-1}^{P} \alpha_{-1}^{P}-\frac{1}{5} \alpha_{-1}^{L} \alpha_{-1}^{L}-\frac{1}{5} \alpha_{-1}^{T} \alpha_{-1}^{T}\right)|0, k\rangle .
$$

Type I ZNS in Eq.(B.4) gives two RZNS

$$
\begin{aligned}
& \left(\alpha_{-2}^{T}-\sqrt{2} \alpha_{-1}^{P} \alpha_{-1}^{T}\right)|0, k\rangle, \\
& \left(\alpha_{-2}^{L}-\sqrt{2} \alpha_{-1}^{P} \alpha_{-1}^{L}\right)|0, k\rangle .
\end{aligned}
$$

RZNS in Eq.(B.6) and Eq.(B.7) correspond to choose $\theta^{\mu}=e^{T}$ and $\theta^{\mu}=e^{L}$ respectively. Note that the norms of Regge "zero-norm" states may not be zero. For instance the norm of Eq.(B.5) is not zero. They are just used to produce Regge stringy Ward identities Eq.(3.29), Eq.(3.27) and Eq.(3.28) in the text.

At the second massive level $k^{2}=-4$, there is a type I scalar ZNS

$$
\begin{aligned}
& {\left[\frac{17}{4}\left(k \cdot \alpha_{-1}\right)^{3}+\frac{9}{2}\left(k \cdot \alpha_{-1}\right)\left(\alpha_{-1} \cdot \alpha_{-1}\right)+9\left(\alpha_{-1} \cdot \alpha_{-2}\right)\right.} \\
& \left.+21\left(k \cdot \alpha_{-1}\right)\left(k \cdot \alpha_{-2}\right)+25\left(k \cdot \alpha_{-3}\right)\right]|0, k\rangle,
\end{aligned}
$$

a symmetric type I spin two ZNS

$$
\left[2 \theta_{\mu \nu} \alpha_{-1}^{(\mu} \alpha_{-2}^{\nu)}+k_{\lambda} \theta_{\mu \nu} \alpha_{-1}^{\lambda \mu \nu}\right]|0, k\rangle, k \cdot \theta=\eta^{\mu \nu} \theta_{\mu \nu}=0, \theta_{\mu \nu}=\theta_{\nu \mu}
$$

where $\alpha_{-1}^{\lambda \mu \nu} \equiv \alpha_{-1}^{\lambda} \alpha_{-1}^{\mu} \alpha_{-1}^{\nu}$ and two vector ZNS

$$
\begin{aligned}
& {\left[\left(\frac{5}{2} k_{\mu} k_{\nu} \theta_{\lambda}^{\prime}+\eta_{\mu \nu} \theta_{\lambda}^{\prime}\right) \alpha_{-1}^{(\mu \nu \lambda)}+9 k_{\mu} \theta_{\nu}^{\prime} \alpha_{-1}^{(\mu \nu)}+6 \theta_{\mu}^{\prime} \alpha_{-1}^{\mu}\right]|0, k\rangle, \theta \cdot k=0,} \\
& {\left[\left(\frac{1}{2} k_{\mu} k_{\nu} \theta_{\lambda}+2 \eta_{\mu \nu} \theta_{\lambda}\right) \alpha_{-1}^{(\mu \nu \lambda)}+9 k_{\mu} \theta_{\nu} \alpha_{-1}^{[\mu \nu]}-6 \theta_{\mu} \alpha_{-1}^{\mu}\right]|0, k\rangle, \theta \cdot k=0 .}
\end{aligned}
$$


Note that Eq.(B.10) and Eq.(B.11) are linear combinations of a type I and a type II ZNS. This completes the four ZNS at the second massive level $M^{2}=4$.

In the Regge limit, the scalar ZNS in Eq.(B.8) gives the RZNS

$$
\left[25\left(\alpha_{-1}^{P}\right)^{3}+9 \alpha_{-1}^{P}\left(\alpha_{-1}^{L}\right)^{2}+9 \alpha_{-1}^{P}\left(\alpha_{-1}^{T}\right)^{2}-9 \alpha_{-2}^{L} \alpha_{-1}^{L}-9 \alpha_{-2}^{T} \alpha_{-1}^{T}-75 \alpha_{-2}^{P} \alpha_{-1}^{P}+50 \alpha_{-3}^{P}\right]|0, k\rangle .
$$

For the type I spin two ZNS in Eq.(B.9), we define $\theta_{\mu \nu}=\sum_{\alpha, \beta} e_{\mu}^{\alpha} e_{\nu}^{\beta} u_{\alpha \beta}$, symmetric and transverse conditions on $\theta_{\mu \nu}$ implies

$$
u_{\alpha \beta}=u_{\beta \alpha} ; u_{P P}=u_{P L}=u_{P T}=0 .
$$

Naively, the traceless condition on $\theta_{\mu \nu}$ implies

$$
u_{P P}-u_{L L}-u_{T T}=0
$$

However, for the reason which will become clear later that one needs to include at least one component $u_{N N}$ perpendicular to the scattering plane and modify Eq.(B.14) to

$$
u_{P P}-u_{L L}-u_{T T}-u_{N N}=0 .
$$

Note that, in the Regge limit, Eq.(B.15) reduces to Eq.(B.14). However, the solutions for Eq.(B.13) and Eq.(B.15) give three RZNS

$$
\begin{aligned}
& \left(\alpha_{-1}^{L} \alpha_{-2}^{L}-\alpha_{-1}^{P} \alpha_{-1}^{L} \alpha_{-1}^{L}\right)|0, k\rangle, \\
& \left(\alpha_{-1}^{T} \alpha_{-2}^{T}-\alpha_{-1}^{P} \alpha_{-1}^{T} \alpha_{-1}^{T}\right)|0, k\rangle, \\
& \left(\alpha_{-1}^{(L} \alpha_{-2}^{T)}-\alpha_{-1}^{P} \alpha_{-1}^{L} \alpha_{-1}^{T}\right)|0, k\rangle,
\end{aligned}
$$

while Eq.(B.13) and Eq.(B.14) give only two RZNS

$$
\left(\alpha_{-1}^{L} \alpha_{-2}^{L}-\alpha_{-1}^{P} \alpha_{-1}^{L} \alpha_{-1}^{L}-\alpha_{-1}^{T} \alpha_{-2}^{T}+\alpha_{-1}^{P} \alpha_{-1}^{T} \alpha_{-1}^{T}\right)|0, k\rangle
$$

and Eq.(B.18). Note that Eq.(B.19) is just a linear combination of Eq.(B.16) and Eq.(B.17). For the high-energy fixed angle calculation in [11, 12], the corresponding extra ZNS will not affect the final result there. The vector ZNS in Eq.(B.10) gives two RZNS

$$
\begin{aligned}
& {\left[6 \alpha_{-3}^{T}-18 \alpha_{-1}^{(P} \alpha_{-2}^{T)}+9 \alpha_{-1}^{P} \alpha_{-1}^{P} \alpha_{-1}^{T}+\alpha_{-1}^{L} \alpha_{-1}^{L} \alpha_{-1}^{T}+\alpha_{-1}^{T} \alpha_{-1}^{T} \alpha_{-1}^{T}\right]|0, k\rangle,} \\
& {\left[6 \alpha_{-3}^{L}-18 \alpha_{-1}^{(P} \alpha_{-2}^{L)}+9 \alpha_{-1}^{P} \alpha_{-1}^{P} \alpha_{-1}^{L}+\alpha_{-1}^{L} \alpha_{-1}^{L} \alpha_{-1}^{L}+\alpha_{-1}^{L} \alpha_{-1}^{T} \alpha_{-1}^{T}\right]|0, k\rangle .}
\end{aligned}
$$


The vector ZNS in Eq. (B.11) gives two RZNS

$$
\begin{aligned}
& {\left[3 \alpha_{-3}^{T}+9 \alpha_{-1}^{[P} \alpha_{-2}^{T]}-\alpha_{-1}^{L} \alpha_{-1}^{L} \alpha_{-1}^{T}-\alpha_{-1}^{T} \alpha_{-1}^{T} \alpha_{-1}^{T}\right]|0, k\rangle,} \\
& {\left[3 \alpha_{-3}^{L}+9 \alpha_{-1}^{[P} \alpha_{-2}^{L]}-\alpha_{-1}^{L} \alpha_{-1}^{L} \alpha_{-1}^{L}-\alpha_{-1}^{L} \alpha_{-1}^{T} \alpha_{-1}^{T}\right]|0, k\rangle .}
\end{aligned}
$$

There are totally 8 RZNS at the mass level $M^{2}=4$.

[1] D. J. Gross and P. F. Mende, Phys. Lett. B 197, 129 (1987); Nucl. Phys. B 303, 407 (1988).

[2] D. J. Gross and J. L. Manes, Nucl. Phys. B 326, 73 (1989). See section 6 for details.

[3] D. Amati, M. Ciafaloni and G. Veneziano, "Superstring Collisions at Planckian Energies,"Phys. Lett. B 197 (1987) 81.

[4] D. Amati, M. Ciafaloni and G. Veneziano, "Classical and Quantum Gravity Effects from Planckian Energy Superstring Collisions," Int. J. Mod. Phys. A 3 (1988) 1615.

[5] D. Amati, M. Ciafaloni and G. Veneziano, "Can Space-Time Be Probed Below The String Size?," Phys. Lett. B 216 (1989) 41.

[6] M. Soldate, "Partial Wave Unitarity and Closed String Amplitudes," Phys. Lett. B 186 (1987) 321.

[7] I. J. Muzinich and M. Soldate, "High-Energy Unitarity of Gravitation and Strings," Phys. Rev. D 37 (1988) 359.

[8] R. C. Brower, J. Polchinski, M. J. Strassler and C. I. Tan, "The pomeron and gauge / string duality," arXiv:hep-th/0603115. JHEP 0712:005,(2007).

[9] Richard C. Brower, Horatiu Nastase, Howard J. Schnitzer, Chung-I Tan, "Analyticity for Multi-Regge Limits of the Bern-Dixon-Smirnov Amplitudes", Nucl.Phys.B822, 301 (2009).

[10] Sheng-Lan Ko, Jen-Chi Lee and Yi Yang, "Patterns of High energy Massive String Scatterings in the Regge regime", JHEP 0906:028,(2009), hep-th/0812.4190.

[11] C. T. Chan and J. C. Lee, Phys. Lett. B 611, 193 (2005). J. C. Lee, arXiv:hep-th/0303012.

[12] C. T. Chan and J. C. Lee, "Zero-norm states and high-energy symmetries of string theory", Nucl. Phys. B 690, 3 (2004).

[13] C. T. Chan, P. M. Ho, J. C. Lee, S. Teraguchi and Y. Yang, "High-energy zero-norm states and symmetries of string theory" Phys. Rev. Lett. 96 (2006) 171601, hep-th/0505035.

[14] C. T. Chan, P. M. Ho, J. C. Lee, S. Teraguchi and Y. Yang, Nucl. Phys. B 725, 352 (2005). 
[15] A. Sagnotti, M. Taronna, "String lessons for higher-spin interactions", Nucl.Phys. B842 (2011) 299-361.

[16] P.C. West, Mod. Phys. Lett.A10(1995)761.

[17] N. Moeller, P.C. West, "Arbitrary four string scattering at high energy and fixed angle", Nucl.Phys. B729 (2005)1.

[18] G.W. Moore,"Finite in all directions", hep-th/9305139 "Symmetries of the bosonic string S-matrix", hep-th/9310026.

[19] J. C. Lee, "New Symmetries Of Higher Spin States In String Theory", Phys. Lett. B 241, 336 (1990); Phys. Rev. Lett. 64, 1636 (1990).

[20] T. D. Chung and J. C. Lee, "Discrete gauge states and W(infinity) charges in c $=1$ 2-d gravity", Phys. Lett. B 350, 22 (1995), hep-th/9412095.

[21] H. C. Kao and J. C. Lee, "Decoupling of degenerate positive-norm states in Witten's string field theory", Phys. Rev. D 67, 086003 (2003), hep-th/0212196. J. C. Lee, "Calculation of zero-norm states and reduction of stringy scattering amplitudes", Prog. Theor. Phys. 114, 259 (2005), hep-th/0302123. C. T. Chan, J. C. Lee and Y. Yang, "Anatomy of zero-norm states in string theory", Phys. Rev. D 71, 086005 (2005), hep-th/0501020.

[22] D. J. Gross, Phys. Rev. Lett. 60, 1229 (1988); D. J. Gross and J. R. Ellis, Phil. Trans. R. Soc. Lond. A329, 401 (1989).

[23] S. He, J.C. Lee, K. Takahashi and Y. Yang, "Massive Superstring Scatterings in the Regge Regime", Phys. Rev. D83 (2011) 066016, hep-th/1001.5392.

[24] J.C. Lee, C.H. Yan and Y. Yang, "High-energy String Scatterings and Signless Stirling Number Identity", SIGMA 8 (2012), 045 ; arXiv:1012.522

[25] Manuel Mkauers, "Summation Algorithms for Stirling Number Identities", Journal of Symbolic Computation, 42(10):948-970 (2007).

[26] L.J.Slater," Confluent Hypergeometric Functions", Cambridge University Press (1960).

[27] Z. Bern, J. J. M. Carrasco and H. Johansson, "New Relations for Gauge-Theory Amplitudes," Phys. Rev. D78, 085011 (2008) [arXiv:0805.3993 [hep-ph]].

[28] N. E. J. Bjerrum-Bohr, P. H. Damgaard and P. Vanhove, "Minimal Basis for Gauge Theory Amplitudes," Phys. Rev. Lett. 103, 161602 (2009) arXiv:0907.1425 [hep-th]].

[29] S. Stieberger, "Open \& Closed vs. Pure Open String Disk Amplitudes," arXiv:0907.2211 [hepth]. 
[30] B. Feng, R. Huang and Y. Jia, "Gauge Amplitude Identities by On-shell Recursion Relation in S-matrix Program," Phys. Lett. B 695, 350 (2011) [arXiv:1004.3417 [hep-th]].

[31] Y. X. Chen, Y. J. Du and B. Feng, "A Proof of the Explicit Minimal-basis Expansion of Tree Amplitudes in Gauge Field Theory," JHEP 1102, 112 (2011) arXiv:1101.0009 [hep-th]].

[32] R. Kleiss and H. Kuijf, "Multi-gluon cross-sections and five jet production at hadron colliders," Nucl. Phys. B 312, 616 (1989).

[33] V. Del Duca, L. J. Dixon and F. Maltoni, "New color decompositions for gauge amplitudes at tree and loop level," Nucl. Phys. B 571, 51 (2000) arXiv:hep-ph/9910563.

[34] Yung-Yeh Chang, Bo Feng, Chih-Hao Fu, Jen-Chi Lee, Yihong Wang and Yi Yang, "A note on on-shell recursion relation of string amplitudes", arXiv:1210.1776 (accepted by JHEP). 